\title{
A model for Gaussian perturbations of graphene
}

Dodson, CTJ

2015

MIMS EPrint: 2015.87

Manchester Institute for Mathematical Sciences

School of Mathematics

The University of Manchester

\footnotetext{
Reports available from: http://eprints.maths.manchester.ac.uk/

And by contacting: The MIMS Secretary

School of Mathematics

The University of Manchester

Manchester, M13 9PL, UK
} 


\title{
A model for Gaussian perturbations of graphene
}

\author{
C.T.J. Dodson \\ School of Mathematics, University of Manchester, M13 9PL, UK \\ ctdodson@manchester.ac.uk
}

\begin{abstract}
Graphene consists nominally of a regular planar hexagonal carbon lattice monolayer. However, its structure experiences perturbations in the presence of external influences, whether from substrate properties, thermal or electromagnetic fields, or ambient fluid movement. Here we give an information geometric model to represent the state space of perturbations as a Riemannian pseudosphere with scalar curvature close to $-\frac{1}{2}$. This would allow the representation of a trajectory of states under a given ambient or process change, so opening the possibility for geometrically formulated dynamical models to link structural perturbations to the physics.
\end{abstract}

Keywords: Graphene, perturbations, information geometry, state space manifold

MSC: 53B20, 62M40, 94A17

\section{Introduction}

A large number of papers have reported micrographic imaging of graphene membranes, revealing the underlying lattice structure with superimposed local variations in topography and induced charges. For example, Hamilton [9] showed typical structural features at several scales in transmission electron micrographs. Costamagna and Dobry [3] studied 2D to 3D transitions in graphene sheets and obtained a correlation between the standard deviation of the out of plane distance and the mean lattice dimension. Meyer et al. [16] showed a transmission electron micrograph of a few-layer graphene membrane and Stolyarova et al. [18] used scanning tunneling microscopy to reveal vertical variations of $0.8 \mathrm{~nm}$ over regions of $20 \mathrm{~nm}$. Couto et al [4] found a linear correlation between reciprocal mobility and the carrier density in all samples tested: thus devices with smaller density fluctuations had greater mobility and this is consistent with the effect of random strain variations in graphene. They were able to confirm the role of random strain fluctuations as an important source of disorder and so account for the above mentioned correlation. Whether this relationship can be linked to a linear correlation between strain standard deviation and mean is an interesting question.

Ishigami et al [11] described a novel heat cleaning method in argon/hydrogen which at the $600 \mathrm{~nm}$ scale reduced the standard deviation of height of graphene on $\mathrm{SiO}_{2}$ from $8 \AA$ to $3 \AA$. The nominal monolayer thickness is $3.4 \AA$ in bulk 
graphite and on $\mathrm{SiO}_{2}$ the layer thickness was measured with an AFM as 4.2A; in air the thickness was $9 \AA$ because of the presence of impurity ambient species at the interface or on the graphene monolayer. From this it suggests that the standard deviation of local thickness of a graphene stratum is of the same order as its mean thickness at the $600 \mathrm{~nm}$ scale. We note also the report by $\mathrm{Xu}$ et al [19] of thermal effects on height fluctuations of freestanding graphene during scanning tunnelling microscopy experiments.

We consider first the simplest situation of tiny Gaussian variations applied to the locations of the carbon atoms in the nominally planar hexagonal graphene structure, which has lattice constants $2.461 \AA$ and $6.708 \AA$. Such, sparse, distributed structural fluctuations would result in behavioural variations, as would departures from planarity caused by substrate carried impurities or other ambient species trapped in between. We believe that these type of fluctuations may be treated as spatially distributed variations and so modelled by probability density functions, which we may formulate as a geometrical space on which transitions or evolution of fluctuation patterns may be represented and linked to observable physical behaviour.

The degeneration of 2D crystal order was simulated by Lucarini through perturbations of the three regular tessellations of the plane: square, hexagonal and triangular, by an increasing spatial Gaussian noise applied to vertices. Physically, there the perturbing spatial noise intensity corresponds to a lattice temperature in the structural symmetry degeneration. The statistical parameters of the evolving changes were analyzed through those of the (convex) cells in the Voronoi tessellations, which are optimal partitions of the space from the given set of generating vertices of the structure. In all cases the gamma distribution was an excellent model for the observed probability density functions of all metric properties: inter-vertex distance, perimeters of polygons, areas of polygons and with the same result for 3D cubic crystal lattices (SC, BCC, FCC) [15], where also the volumes of polyhedra followed gamma distributions. With the onset of noise, quite quickly the three $2 \mathrm{D}$ tessellations became indistinguishable. Similar results were found also for perturbations of the three 3D cubic crystal lattices [15, 5]. Lucarini [14] suggested that such an approach could be made to model the structure of graphene.

The results of Lucarini's simulations of $2 \mathrm{D}$ and $3 \mathrm{D}$ crystal disordering were put into an information geometric framework [5] by means of which the space of perturbations was represented by a Riemannian manifold of gamma probability density functions provided with the Fisher information metric [2]. That allowed the representation of the tessellation-constrained degeneration down to the Poisson Voronoi limit.

Remark: Here we are not concerned with such large scale degeneration as in [13] but with very small variations in the hexagonal structure of graphene, which might resemble the early stages of the Gaussian perturbations of a hexagonal crystal lattice. The small scale of such perturbations mitigates the concern that applying the same Gaussian variation to the location of carbon atoms for graphene and to the 'mother'point for Voronoi tessellation could result in different structures. We believe that at the scale envisaged this difference would 
be small, but certainly worth investigating through simulations, and if possible experimentally. A further concern in the case of graphene was that the dynamical behaviour of the network of carbon atoms could possibly bring about different distributional properties from the case of 'static'Voronoi tessellations. Again this is worthy of investigation but at small scales the dynamical variations might be expected to yield oscillations that centred on the mean static case.

The application of the information geometric methodology to real data depends on estimating the mean and variance of the distributions of e.g. hexagon perimeters in an ideal planar model but additionally obtaining also distributions of out of plane distances for vertices if non-planar fluctuations are present. Such data could then be used to represent the distributions of fluctuations from a regular planar hexagonal crystal, either using individual lengths or combinations thereof. In any case we expect that the distributions involved will be gamma and that variations in the influences causing the fluctuations will move the distribution about in the space of gamma distributions. We outline how an information geometric approach may help model the behaviour through the provision of a geometrically formulated natural state space on which to represent physical influences. It may be that the bivariate gamma distribution discussed with applications in [2] could be used as the model if correlated horizontal and vertical fluctuations are observed in graphene.

The gamma distribution, which seems to be encountered in many naturally occurring processes [2], can be characterised by the following uniqueness theorem:

Theorem $1.1([\mathbf{1 2}, 10])$ For independent positive random variables with a common probability density function $f$, having independence of the sample mean and the sample coefficient of variation is equivalent to $f$ being the gamma distribution.

A proof was given by Hwang and $\mathrm{Hu}[10$ ] but the result seems to have been known earlier and in [6] we gave a proof partly based on the 1954 article by Laha [12].

The family of gamma distributions with random variable $x$ in event space $\Omega=\mathbb{R}^{+}$has a collection of probability density functions given by

$$
\left\{f(x ; \mu, \kappa)=\left(\frac{\kappa}{\mu}\right)^{\kappa} \frac{x^{\kappa-1}}{\Gamma(\kappa)} e^{-x \kappa / \mu} \mid \mu, \kappa \in \mathbb{R}^{+}\right\} \equiv \mathbb{R}^{+} \times \mathbb{R}^{+},
$$

and $\Gamma$ is the gamma function. The gamma probability density functions (1) depend smoothly on parameters $\mu, \kappa \in \mathbb{R}^{+}$. The mean is $E[x]=\mu$, the variance is $E\left[x^{2}\right]-E[x]^{2}=\sigma^{2}=\mu^{2} / \kappa$ and so we see that $\frac{1}{\sqrt{\kappa}}$ is the constant of proportionality between the standard deviation and the mean, which reflects the result in Theorem 1.1 .

Given a set of identically distributed, independent data values $X_{1}, X_{2}, \ldots, X_{n}$, the 'maximum likelihood' or 'maximum entropy' parameter values $\hat{\mu}, \hat{\kappa}$ for fitting the gamma distribution (11) are computed in terms of the mean and 
mean logarithm of the $X_{i}$ by maximizing the likelihood function

$$
L i k_{f}(\mu, \kappa)=\prod_{i=1}^{n} f\left(X_{i} ; \mu, \kappa\right) .
$$

Taking the logarithm and setting the gradient to zero we obtain

$$
\begin{aligned}
\hat{\mu} & =\bar{X}=\frac{1}{n} \sum_{i=1}^{n} X_{i} \\
\log \hat{\kappa}-\frac{\Gamma^{\prime}(\hat{\kappa})}{\Gamma(\hat{\kappa})} & =\log \bar{X}-\frac{1}{n} \sum_{i=1}^{n} \log X_{i} \\
& =\log \bar{X}-\overline{\log X}
\end{aligned}
$$

We note that the distribution of a sum of independent gamma random variables is itself gamma distributed. The special case $\kappa=1$ in (1) corresponds to the situation of the random or Poisson process with mean $\mu$ (and so also $\sigma=\mu$ ) then the distribution of size of inter-event spaces is exponential, the unique distribution with unit mean having maximum entropy-that is least constraints.

In fact, the gamma distribution has an essential generalising property of the exponential distribution since in particular it represents inter-event distances for generalisations of the Poisson process to a 'censored' Poisson process. Indeed, for integer $\kappa=1,2, \ldots$, (1) models a process that is Poisson but with intermediate events removed to leave only every $\kappa^{\text {th }}$. Formally, the gamma distribution is the $\kappa$-fold convolution of the exponential distribution, called also the Pearson Type III distribution. The Chi-square distribution with integer $n=2 \kappa$ degrees of freedom models the distribution of a sum of squares of $n$ independent random variables all having the Gaussian distribution with zero mean and standard deviation $\sigma$; this is a gamma distribution with mean $\mu=n \sigma^{2}$ for integer $\kappa=1,2, \ldots$

We might expect that the horizontal structural fluctuations in graphene are small-scale rare events in a random process, such as a perturbation of a Poisson spatial process. A wide range of near-Poisson processes is discussed in terms of the information geometry of the gamma family in [2]. In the context of small structural fluctuations in graphene there may be an interpretation of the anticipated very high values of $\kappa$ compared with unit mean in terms of the censored Poisson process corresponding to rare events, for example, small and rare variations in hexagon perimeter or area. Accordingly, here we investigate the properties of the gamma distribution for possible modeling of graphene, if observational data becomes available then other choices may arise, including bivariate models for vertical and horizontal variability.

Whereas the nominal monolayer thickness of graphene is $3.4 \AA$ in bulk graphite, on $\mathrm{SiO}_{2}$ it is $4.2 \AA$ and in air the impurities increased the thickness to $9 \AA$. At the $600 \mathrm{~nm}$ scale the standard deviation of height was of similar size to the mean thickness [11], which suggests that the coefficient of variation is unity and the vertical process is approximately exponential. 


\section{Information geometry of the gamma distribution}

For any smooth family of probability density functions

$$
\left\{p_{\theta} \mid \theta \in \Theta \subseteq \mathbb{R}^{n}\right\}
$$

defined on some fixed event space $\Omega$ (typically $\mathbb{R}, \mathbb{R}^{+}$or products thereof) the covariance matrix $\left[g_{i j}\right]$ is the expectation of the matrix of derivatives of the loglikelihood function $l=\log p_{\theta}$, with respect to parameters $\left(\theta^{i}\right)$. This is positive definite and hence defines a Riemannian metric on the smooth $n$-manifold of probability density functions with coordinates $\left(\theta^{i}\right)$.

The components of the Riemannian metric are given by the expectation of the covariance matrix of gradients of the log-likelihood function $l$ with respect to the $n$ parameters $\left(\theta^{i}\right)[1,2]$ :

$$
\left[g_{i j}\right]=E\left(\frac{\partial l}{\partial \theta^{i}} \frac{\partial l}{\partial \theta^{j}}\right)=\left[\int_{\Omega} p_{\theta}\left(\frac{\partial l}{\partial \theta^{i}} \frac{\partial l}{\partial \theta^{j}}\right)\right],
$$

or equivalently, under mild regularity conditions [17],

$$
\left[g_{i j}\right]=E\left(\frac{\partial^{2} l}{\partial \theta^{i} \partial \theta^{j}}\right)=\left[\int_{\Omega} p_{\theta}\left(\frac{\partial^{2} l}{\partial \theta^{i} \partial \theta^{j}}\right)\right]
$$

with arc length function

$$
d s^{2}=\sum_{i, j} g_{i j} d \theta^{i} d \theta^{j}
$$

The family (4) is called an exponential family if the $p_{\theta}$ admit expression in terms of functions $\left\{C, F_{1}, \ldots, F_{n}\right\}$ on $\Omega$ and a function $\varphi$ on $\Theta$ as:

$$
p_{\theta}(x)=e^{\left\{C(x)+\sum_{i} \theta_{i} F_{i}(x)-\varphi(\theta)\right\}},
$$

then these $\left(\theta_{i}\right)$ are its natural parameters, and $\varphi$ is the potential function. By integrating (7) over $\Omega$, then using

$$
\int p(x ; \theta) d x=1, \quad \text { on the left }
$$

we take logs and obtain:

$$
\varphi(\theta)=\log \int e^{\left\{C(x)+\sum_{i} \theta_{i} F_{i}(x)\right\}} d x .
$$

For the gamma distribution, it can be seen that $(\nu=\kappa / \mu, \kappa)$ are natural parameters and its potential function is

$$
\varphi(\nu, \kappa)=\log \Gamma(\kappa)-\kappa \log \nu .
$$

In these coordinates the components of the metric are given by

$$
\left[g_{i j}\right](\nu, \kappa)=\left[\begin{array}{cc}
\frac{\kappa}{\nu^{2}} & -\frac{1}{\nu} \\
-\frac{1}{\nu} & \frac{d^{2}}{d \kappa^{2}} \log (\Gamma)
\end{array}\right]
$$


In such cases the $n$-manifold of probability density functions can be represented by a natural affine immersion in $\mathbb{R}^{n+1}$ via

$$
h:(\theta) \in \mathbb{R}^{n} \mapsto((\theta), \varphi(\theta)) \in \mathbb{R}^{n+1} .
$$

So the natural representation of the manifold of gamma distributions $M$ in $\mathbb{R}^{3}$ is

$$
h: M \rightarrow \mathbb{R}^{3}:(\nu, \kappa) \mapsto(\nu, \kappa, \log \Gamma(\kappa)-\kappa \log \nu)
$$

This is convenient for visualising and metrising curves that depict trajectories in the space of gamma distributions [2]; observational data for graphene structures under varying external conditions could then be visualised and correlated with the physics.

For an exponential family $(7)$ there is a simpler method to compute the information metric (5) from the $\log$-likelihood function $l(\theta, x)=\log p_{\theta}(x)$ :

$$
\partial_{i} l(\theta, x)=F_{i}(x)-\partial_{i} \varphi(\theta)
$$

and

$$
\partial_{i} \partial_{j} l(\theta, x)=-\partial_{i} \partial_{j} \varphi(\theta), \text { which is independent of } x .
$$

Then the information metric $g$ on the $n$-dimensional space of parameters $\Theta \subset$ $\mathbb{R}^{n}$, equivalently on the set $\left\{p_{\theta} \mid \theta \in \Theta \subset \mathbb{R}^{n}\right\}$, has components:

$$
\left[g_{i j}\right](\theta)=-\int_{\Omega}\left[\partial_{i} \partial_{j} l(\theta, x)\right] p_{\theta}(x) d x=\partial_{i} \partial_{j} \varphi(\theta)=\left[\varphi_{i j}\right](\theta) .
$$

The gamma distribution has a surprisingly tractable information geometry [1. [2, and the Riemannian metric in the 2-dimensional manifold $(M, g)$ of gamma distributions (1) is easily computed from the definition (5) in $(\mu, \kappa)$ coordinates:

$$
\left[g_{i j}\right](\mu, \kappa)==\left[\begin{array}{cc}
\frac{\kappa}{\mu^{2}} & 0 \\
0 & \frac{d^{2}}{d \kappa^{2}} \log (\Gamma)-\frac{1}{\kappa}
\end{array}\right] .
$$

So the coordinates $(\mu, \kappa)$ yield an orthogonal basis of tangent vectors, which is useful in calculations because then the arc length function in $M$ is simply

$$
d s^{2}=\frac{\kappa}{\mu^{2}} d \kappa^{2}+\left(\left(\frac{\Gamma^{\prime}(\kappa)}{\Gamma(\kappa)}\right)^{\prime}-\frac{1}{\kappa}\right) d \kappa^{2}=\frac{\kappa}{\mu^{2}} d \kappa^{2}+\left(\psi^{\prime}(\kappa)-\frac{1}{\kappa}\right) d \kappa^{2}
$$

where $\psi^{\prime}(\kappa)=\left(\frac{\Gamma^{\prime}(\kappa)}{\Gamma(\kappa)}\right)^{\prime}$.

Such a manifold with the Levi-Civita metric connection (cf. eg [7]) is a pseudosphere, with its negative scalar curvature given by [2]:

$$
R(\kappa)=\frac{\psi^{\prime}(\kappa)+\kappa \psi^{\prime \prime}(\kappa)}{4\left(\kappa \psi^{\prime}(\kappa)-1\right)^{2}} \quad \text { so }-\frac{1}{2}<R(\kappa)<-\frac{1}{4}
$$

from which it follows that $R(\kappa) \rightarrow-\frac{1}{2}$ as $\kappa \rightarrow \infty$.

In the context of horizontal structural fluctuations of inter-vertex distances or hexagon perimeter length in graphene with a unit mean, we expect the standard deviation $\sigma=\frac{\mu}{\sqrt{\kappa}}<<1$ hence it follows that $\kappa>>1$ and the scalar curvature 17 will be close to $-\frac{1}{2}$. 


\section{$3 \quad$ A state space for graphene structural fluctuations}

For the standard deviation of, say, hexagon perimeters, $\sigma(P)=\mu(P) / \kappa$, to be between $1 \%$ and $10 \%$ of the mean, for example, we have a range of $100<\kappa<$ 10000 for the relevant gamma distributions. A propos the characterisation of the gamma distribution in Theorem 1.1. Couto et al [4] confirmed the role of random strain fluctuations as an important source of disorder and so accounted for the observed correlation between reciprocal mobility and the carrier density in all samples tested. Whether this relationship can be linked to a linear correlation between strain standard deviation and mean strain is an interesting question from the point of view of a model using a gamma distribution.

Formulating the family of gamma probability density functions as a Riemannian manifold in natural coordinates we can use geometric methods to describe the progress of fluctuation changes on property behaviour and provide a graphical representation through a natural affine immersion in $\mathbb{R}^{3}$.

Given a differential equation on the manifold to represent an evolution of fluctuations, its integral curves would yield trajectories for the structural state. On a space of probability density functions there is always one natural vector field, arising from the gradient field of the entropy function. This differential equation and its integral curves in the manifold of gamma probability density functions represent progress of random disordering (maximising entropy) as intensity of disturbance increases. So this field may represent well enough the initial development of structural changes with increasing fluctuation intensity in graphene. Then the physical manifestation in properties or associated behaviour might be linked to the effect of fluctuation intensity.

The Shannon entropy of the gamma family (1) is given in both sets of coordinates by:

$$
\begin{aligned}
S_{f}(\nu, \kappa) & =-\int_{0}^{\infty} f \log f d x: \mathbb{R}^{2+} \rightarrow \mathbb{R} \\
(\nu, \kappa) & \mapsto \kappa-(\kappa-1) \psi(\kappa)+\log (\Gamma(\kappa))-\log (\nu) \\
(\mu, \kappa) & \mapsto \kappa-(\kappa-1) \psi(\kappa)+\log (\Gamma(\kappa))-\log (\mu / \kappa)
\end{aligned}
$$

where $\psi=\frac{\Gamma^{\prime}}{\Gamma}$ is the digamma function. The entropy gradient vector field is

$$
\begin{aligned}
& \nabla S_{f}(\nu, \kappa)=\left\{-\frac{1}{\nu}, 1-(\kappa-1) \psi^{(1)}(\kappa)\right\} \\
& \nabla S_{f}(\mu, \kappa)=\left\{-\frac{\kappa}{\mu}, 1-(\kappa-1) \psi^{(1)}(\kappa)\right\}
\end{aligned}
$$

and an unconstrained degeneration of order would follow its integral curves down to $\kappa=1$. In fact of course we do not have unconstrained disordering for graphene because we must retain the hexagonal structure, so we would expect a slightly less steep descent with increasing fluctuation intensity, ie with increasing noise level $a$ in the terms of the hexagonal crystal lattice simulations. For the hexagonal simulation [15] the distribution of perimeters of 
the hexagons followed a gamma distribution; the mean perimeter was $\bar{P} \approx 0.65$ and the standard deviation of the perimeter was given approximately by

$$
\sigma(P) \approx \frac{a}{\sqrt{6}} \text { for } 0<\mathrm{a}<0.5
$$

In the $2 \mathrm{D}$ crystal cases simulated by Lucarini [13] the hexagonal structure was stable under small perturbations but the square and triangular structures were not stable. However, with the onset of noise, quite quickly all three possible tessellations became indistinguishable above noise $a \approx 0.5$. With intense noise they converged to the 2D Poisson-Voronoi tessellations, for which exact analytic results are known [8]. The limiting values were $\kappa \approx 16$ for the perimeter of polygons and $\kappa \approx 3.7$ for areas. Hence in our context, for fluctuations of order $1 \%$ of the mean, say, $\sigma(P) \approx 0.01 \mu(P) \approx 0.0065$, which corresponds to the noise amplitude $a \approx 0.016$ in $[13$.

\section{Discussion}

Ideally, we would like experimental data on vertical and horizontal spatial variations in graphene. Certainly there is little in the published literature on the distribution and scale of structural fluctuations that have been observed. Until more details become available we take the view that a representation of spatially distributed fluctuations could provide a stimulus to generate suitable data; when such data is available the model here is easily adapted, and could incorporate a bivariate distribution for vertical and horizontal variability. In due course it may be that a product distribution of vertical and horizontal variations may seem more appropriate, then, for example the bivariate gamma distribution described with applications in [2] may be appropriate. Such a bivariate model will be considered elsewhere.

If variations other than Gaussian perturbations better represent the fluctuations in graphene, then suitable information geometric models could use appropriate other models. For the present, we have some quantitative clues to the possible parameters of a model for fluctuations in graphene structure. These come from computer simulations of Gaussian perturbations of hexagonal crystal lattices [13, 15], from limited experimental data on spatial variability of vertical deviations [16, 11, 18], and from observed correlation of vertical standard deviation with mean lattice dimension [3] which confirmed the role of random strain variations in graphene.

Whereas the nominal monolayer thickness of graphene is $3.4 \AA$ in bulk graphite, on $\mathrm{SiO}_{2}$ it is $4.2 \AA$ and in air the impurities increased the thickness to $9 \AA$. At the $600 \mathrm{~nm}$ scale the standard deviation of height was of similar size to the mean thickness, which suggests that the coefficient of variation is unity and the vertical process is approximately exponential and hence arises from a Poisson spatial process. The spatial variations in the horizontal structure are, however, expected to have a standard deviation much smaller than the mean for local hexagon perimeter. 
The information geometry of the gamma manifold turns out to be that of a Riemannian pseudosphere [2] with scalar curvature $R(\kappa)$ given in (17), from which it follows that $R(\kappa) \rightarrow-\frac{1}{2}$ as $\kappa \rightarrow \infty$. In the context of structural fluctuations of inter-vertex distances or hexagon perimeters in graphene with a unit mean, we expect the standard deviation $\sigma=\frac{\mu}{\sqrt{\kappa}}<<1$ hence it follows that $\kappa>>1$ and the scalar curvature 17 will be close to $-\frac{1}{2}$. There is a natural embedding of this space as a curved surface in $\mathbb{R}^{3}[2$, and trajectories of structural changes can be represented on this surface as curves to which any observed physical features could be attached.

Acknowledgement The author is grateful to an anonymous reviewer who made the points addressed in the Remark on the second page.

\section{References}

[1] S-I. Amari and H. Nagaoka. Methods of Information Geometry, American Mathematical Society, Oxford University Press, 2000.

[2] K. Arwini and C.T.J. Dodson. Information Geometry Near Randomness and Near Independence. Lecture Notes in Mathematics. Springer-Verlag, New York, Berlin, 2008, Chapter 9 with W.W. Sampson, Stochasic Fibre Networks pp 161-194.

[3] S. Costamagna and A. Dobry. From the 2D graphene honeycomb lattice to 1D nanoribbons: dimensional crossover signals in the structural thermal fluctuations. Phys. Rev. B 83, 233401 (2011) 1-4. cf. also arXiv:1104.3763v1

[4] Nuno J. G. Couto, Davide Costanzo, Stephan Engels, Dong-Keun Ki, Kenji Watanabe, Takashi Taniguchi, Christoph Stampfer, Francisco Guinea, and Alberto F. Morpurgo. Random Strain Fluctuations as Dominant Disorder Source for High-Quality On-Substrate Graphene Devices Phys. Rev. X 4, 041019 (2014) 1.-13.

[5] C.T.J. Dodson. On the entropy flows to disorder. In C. H. Skiadas and I. Dimotikalis, (Eds.), Chaotic Systems: Theory and Applications World Scientific, Singapore, 2010 pp 75-84. Cf. also http://arxiv.org/abs/0811.4318

[6] C.T.J. Dodson. Some illustrations of information geometry in biology and physics Chapter 13 in Handbook of Research on Computational Science and Engineering: Theory and Practice Eds. J. Leng, W. Sharrock, IGI-Global, 2012. pp 287-315.

[7] C.T.J. Dodson and T. Poston. Tensor Geometry Graduate Texts in Mathematics 130, Second edition, Springer-Verlag, New York, 1991.

[8] J.-S. Ferenc and Z. Néda. On the size distribution of Poisson Voronoi cells. Physica A: Statistical Mechanics and its Applications, 385, 2 (2007) 518-526. 
http://arxiv.org/PS_cache/cond-mat/pdf/0406/0406116v2.pdf (2008).

[9] C.E. Hamilton and A.R. Barron. Graphene. In Nanomaterials and Nanotechnology Ed. A.R. Barron, Rice University, 2013. http://cnx.org/content/m29187/latest/?collection=col10700/latest

[10] T-Y. Hwang and C-Y. Hu: On a characterization of the gamma distribution: The independence of the sample mean and the sample coefficient of variation. Annals Institute Statistical Mathematics 51(4) 749-753 (1999).

[11] Masa Ishigami, J.H. Chen, W.G. Cullen, M.S. Fuhrer, and E. D. Williams. Atomic Structure of Graphene on SiO2. NANO LETTERS 7,6 (2007) 1643-1648.

[12] R.G. Laha: On a characterization of the gamma distribution. Ann. Math. Stat. 25 (1954) 784-787.

[13] Valerio Lucarini. From Symmetry Breaking to Poisson Point Processes in 2D Voronoi Tessellations: the Generic Nature of Hexagons. J. Stat. Phys. 130 (2008) 1047-1062.

[14] Valerio Lucarini. Symmetry-break in Voronoi tessellations. Symmetry 1 (2009) 1-33. doi:10.3390/sym1010021

[15] Valerio Lucarini. Three-dimensional Random Voronoi Tessellations: From Cubic Crystal Lattices to Poisson Point Processes. J. Stat. Phys. 134(1) (2009) 1047-1062.

[16] J.C. Meyer, A.K. Geim, M.I. Katsnelson, K.S. Novoselov, T.J. Booth and S. Roth. The structure of suspended graphene sheets. Nature Letters 446 (1 March 2007) 60-63. doi:10.1038/nature05545

[17] S.D. Silvey. Statistical Inference Chapman and Hall, Cambridge, 1975.

[18] E. Stolyarova, K.T. Rim, S. Riu, J. Maultzsch, P. Kim, L.E. Brus, T.F. Heinz, M.S. Hybertsen and G.W. Flynn. High-Resolution Scanning Tunneling Microscopy Imaging of Mesoscopic Graphene Sheets on an Insulating Surface. Proc. Nat. Acad. Sc. 104, 22 (2007) 9209-9212. doi:10.1073/pnas.0703337104

[19] P. Xu, M. Neek-Amal, S. D. Barber, J. K. Schoelz, M. L. Ackerman, P. M. Thibado, A. Sadeghi and F. M. Peeters. Unusual ultra-low-frequency fluctuations in freestanding graphene. Nature Communications 28 April (2014) 1-7. DOI: $10.1038 /$ ncomms 4720 